\title{
The Compound Spectral Indices of Human Stress
}

\author{
Petr Kloucek, Armin Von Gunten \\ CAMPsyN, SUPPA, Hôpital de Cery, Lausanne, Switzerland \\ Email:petr.kloucek@iCloud.com,petr.kloucek@chuv.ch,armin.von-gunten@chuv.ch
}

How to cite this paper: Kloucek, P. and Von Gunten, A. (2018) The Compound Spectral Indices of Human Stress. Applied Mathematics, 9, 1378-1394.

https://doi.org/10.4236/am.2018.912090

Received: August 3, 2018

Accepted: December 25, 2018

Published: December 28, 2018

Copyright $(92018$ by authors and Scientific Research Publishing Inc. This work is licensed under the Creative Commons Attribution International License (CC BY 4.0).

http://creativecommons.org/licenses/by/4.0/

\begin{abstract}
Temporally fine-grained and objective measures of mental states or their surrogate states are desperately needed in clinical psychiatry. Stress, both acute and especially chronic stress, is an important mental and physiological state observed in many mental disorders. It is a potential precipitant of acute psychiatric decompensations, be they anxious, affective, psychotic, or behavioural. Thus, being able to objectively follow stress or its surrogate parameters over time in a clinician-friendly way would help predict and prevent decompensations and monitor subsequent treatment success. Thus, we introduce the Compound Spectral Stress Indices (CSSI) that are derived from sensing data of various physiological and physiological and behavioural parameters we use as surrogate stress measures. To obtain the CSSI we use a hierarchical approach provided by adaptability, congruency and derived stress coefficient matrices. Adaptability is defined as a macroscopic characterisation of physiological and physiological and behavioural performance constructed as a product of the total variation of time-segmented complexity indices multiplied by the frequency of the time-varying distribution of complexity indices of the measured physiological or physiological and behavioural parameters, where complexity is expressed in terms of the Hurst exponent. Congruency is expressed by a constant characterising a demand-resource balance and it is then expressed in the form of a stress coefficient matrix. The CSSI is given by the spectral distance of the stress coefficient matrices from the ideal demand-resource matrix.
\end{abstract}

\section{Keywords}

Stress, Physiological and Behavioural Complexity, Surrogate

Data, Self-Similar Normally Distributed Processes,

Hurst Exponent 


\section{Introduction}

Real, symbolic, or imagined stimuli perceived as threatening by a person represent a stress that elicits a system response. The system response is physiological and physiological and behavioural in nature and aims at discarding the threat. When a threat occurs, an alarm sets off an adaptive response or adaptation that leads to resolution and returns to the initial equilibrium if the stressor subsides or is discarded or to a state of chronic distress if the stressor persists. The initial physiological and behavioural response is governed by the orthosympathetic nervous system resulting in multiple neurotransmitter, immunological and brain changes [1], and a physiological and behavioural response resulting in fight, flight or freezing. After the adaptive response the physiological and behavioural features return to the previous homeostasis, or a new and possibly pathological equilibrium if the stressor persists for longer periods of time resulting in chronic stress.

We view human stress as a state when demands due to stressors-momentarily, in a form of positive or negative acute stress, or permanently, in a form of chronic stress-challenge and sometimes exceed, in the case of either overwhelming acute or chronic stress, the brain's capabilities to cope with the demands.

The physiological and behavioural response to stress and its return to normal homeostasis or a new possibly pathological equilibrium can be objectively measured. Some of these measures require invasive techniques determining, e.g., neurotransmitter, endocrine, or brain perfusion changes, while others are noninvasive including measures of heart rate, respiratory rate, skin conductance, or movement among others [2] [3]. Invasive techniques allow only for measures that are temporarily coarse-grained. The non-invasive techniques allow for continuous observations. Stress and stress responses leading or not to new equilibria are temporal phenomena. None of the available measures are, however, synonymous with the stress response itself as they measure specific aspects related to stress situations and reactions. Hence, continuous observational techniques are of paramount importance to measure stress. Thus, measuring several of these parameters over time appears as a reasonable approximate measure of the more complex overall phenomenon of stress. Thus, we rely on physiological and physiological and behavioural measures as surrogate data for stress or, in other words, its bodily expression. These surrogate data can be recorded using bodyattached sensors [4] [5] [6] [7].

The purpose of this paper is to translate the assessment of stress using surrogate data into a mathematical model that can ultimately be used in clinical settings to objectively quantify the stress a human subject experiences. In other words, we strive to introduce an objective measure of stress and its severity using a combination of sensing and recording of complex time-series of physiological and behavioural measures and their posterior mathematical complexity analysis to arrive at the Compound Spectral Stress Index. 
The mathematical part of the paper starts with the introduction of Adaptability in the subsection with the corresponding title. Then, the notion of the Stress Coefficient Matrix (SCM) is introduced in the subsection Stress Coefficient Matrix. SCM relates two affine-images of time-discrete surrogate data that are self-similar normally distributed stochastic processes. The stress coefficients assembled in SCM yields congruency, c.f., subsection Congruency. The notion of congruency is constructed to reflect the external demands/regulatory capacities of the human nervous system. The notion of physiological and behavioural congruency allows defining an ideal demands/response SCM. Defining an ideal SCM leads immediately to the definition of the Compound Spectral Stress Index (CSSI) that is represented by the spectral distance of the actual complexity-based SCM from the ideal system response, c.f., subsection Compound Spectral Stress Index. CSSI is represented by a single positive real number. We propose that this index would play a similar role for stress as temperature body measurements in general medicine, but taking instead the results of the physiological and behavioural complexity analysis instead of just one or more averaged physiological and behavioural readings [8].

Multiple physiological and behavioural parameters change over time driven by stress and stress-related responses. Consequently, these parameters can be combined in various ways yielding different CSS Indices addressing different structures of observable stress pronunciations [9].

For the illustrative purpose of this paper, we use physiological (heart rate, blood oxygenation) and motor (steps frequency) features as physiological and behavioural surrogate markers of stress, of which we know that they have by far the largest variance among all the sensory readings we have investigated previously [10] [11]. Specifically, the recordings and analyses of eight healthy individuals during normal working days, sleep and exercise are shown for illustrative purposes only.

\section{Quantitative Methods}

\subsection{Measuring Surrogate Data of Stress}

Indications of stress can be measured using self-rated questionnaires, temporarily coarse-grained invasive physiological means, such as blood-sampled biomarkers, or temporarily fine-grained or continuous physiological or physiologi$\mathrm{cal}$ and behavioural means [12]. These approaches are not mutually exclusive and reveal that stress is a multidimensional construct. We focus on continuous physiological and behavioural measures as they are both objective compared to the questionnaire approach, that is subjective, and capture the short-term temporal dynamics of the stress response as opposed to coarse-grained measures such as blood sampling. Another direct advantage is that the presented approach is not invasive.

However, there are at least two fundamental problems with measuring stress. First, stress is not measurable in the sense of physics as it has no physical units. 
Second, indirect stress indicators such as self-reporting, bodily fluid analyses [13], or multi-dimensional complexity analysis [10], require micro-to-macroscopic translations that are not a priori invertible. We use the notion of invertibility to indicate, for example, that even if we could easily detect microscopic structures by the presence of certain molecules, a process linked to macroscopic observable stress states might not induce such states under certain circumstances.

Let us consider an example. The corticotropic stress response is one of the pronunciations of stress, among many others [1], and part of the regulatory system response. Nevertheless, it is neither the stress itself nor does it have the same unit. This holds true for any of the measurables that we therefore call surrogate data. Furthermore, the attempt to quantify stress faces the difficulty that the various surrogate data are situated at vastly different scales: from microscopic chemical reactions all the way up to physiological changes such as heart rate or skin conductance and further to macsroscopic topological measurements such as torso motions or positional changes measurable when the stress response implies flight-or-fight, let alone the subjective psychological phenomena that accompany stress.

We accept that it is not necessary to express stress in physical units for its pronunciations can be measured by its effects on physiology and behaviour. These effects expressed via surrogate data are measurable physical quantities.

The neurochemical stress-related changes are not directly amenable to continuous and non-invasive measuring. Thus, we would rather focus on macroscopic stress equivalents, i.e., physiological and behavioural measures, that can be measured non-invasively over time. We have provided evidence that some surrogate physiological and behavioural data, including heart rate, skin blood perfusion, blood oxygenation, skin temperature, movement and steps frequency obtained using body-attached sensors exhibit approximately normally distributed and self-similar time series [10]. This allows for complexity analysis of such data and for simultaneous multimodal projections in place of observational statistics. We introduce an application of spectral theory to implement a compound analysis of multiple macroscopic characterisations of physiological and behavioural surrogate data.

\subsection{Spectral Characterisation of Stress}

The presented concepts of the stress coefficients and the compound stress indices are as follows. We introduce adaptability, c.f., subsection Adaptability, that is a product of complexity total variation and frequency reflecting both the qualitative and quantitative changes of the time-dependent acquired data.

Further, we introduce a notion of stress coefficient based on adaptability in subsection Stress Coefficient. It is a constant of which we propose that it may reflect the relation between stressors and response based upon adaptability and the environmental demands [14].

Adaptability is measured through various stress coefficients that can be compared and their congruency determined, c.f., subsection Congruency. Congru- 
ency is constructed to indicate that reactivity and demands, in other words adaptibility, are at equilibrium. An example of a stress coefficient can be produced by comparing adaptability of, e.g., heart rate with steps frequency.

We present the structure of the theory at Figure 1.

The fundamental principle we adopt is that stress, not directly measurable in the sense of physics, can be constitutively modelled by multidimensional Euclidian spaces derived from complexity analysis and its consequent indexing of macroscopic surrogate data.

There is a consequence of the outlined principle. We use physical quantities on an intermediate level formed by sensory data acquisitions. These time-series have associated SI units. Their projection on the complexity indices removes the units disparity.

Our approach is designed to provide a deeper understanding of physiological and behavioural patterns, an understanding that cannot be obtained using standard observational statistics such as, e.g., mean, variance and correlation of the raw data. An illustration of the presented approach is shown at Figure 2.

\section{Quantitative Methods Theory}

We do not measure stress or demands at the data collection levels. We measure reactions of an organism of which we assume that they can provide a constitutive model of stress. The hierarchical constitutive mathematical model, described in subsections Adaptability, Stress Coefficient, Congruency, and Compound Spectral Stress Index, is as follows.

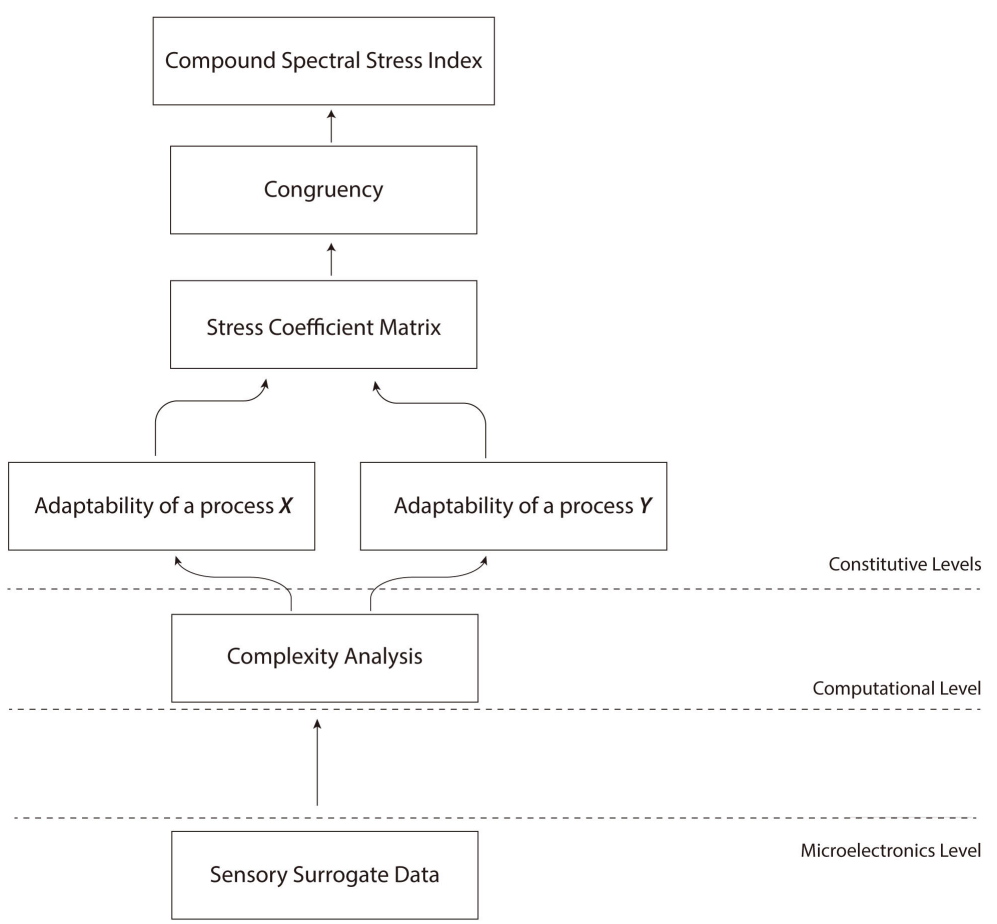

Figure 1. The structure of the presented theory yields the spectral index. The arrows indicate an order in which these indices are built. 


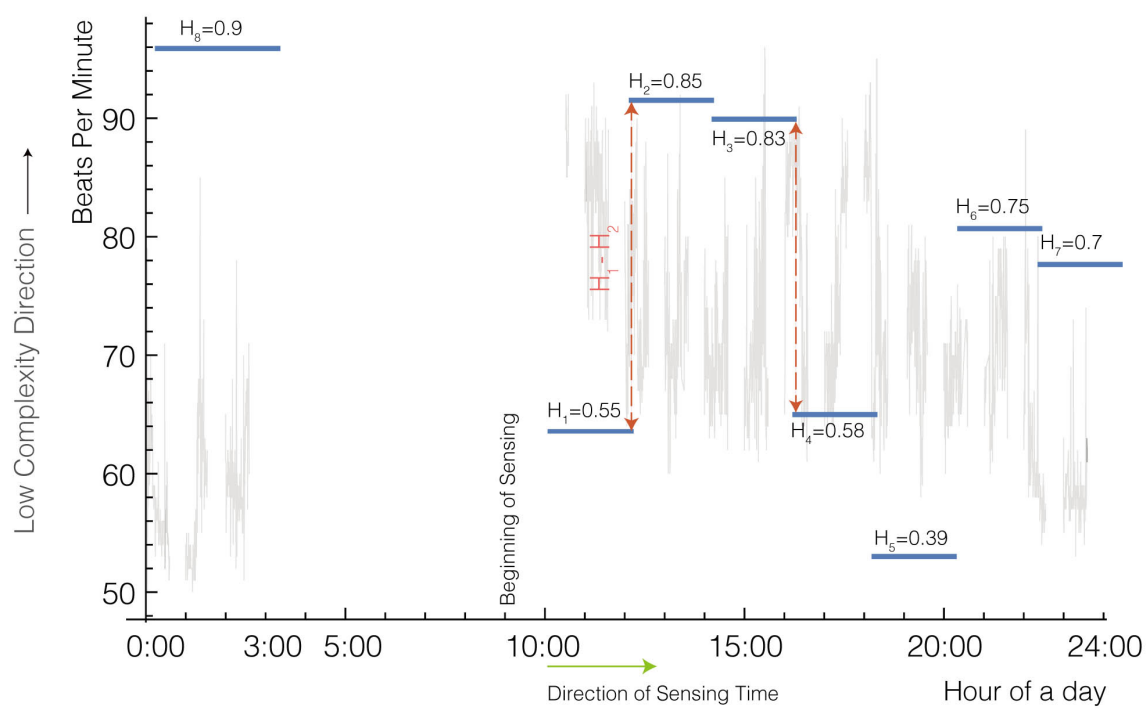

Figure 2. Heart rate data segmentation, c.f., [10]. The step function (blue piece-wise constant function) correlates only with the time axis. It represents values of the Hurst exponents computed from data points corresponding to about fifteen hours segmented to two hours time intervals. The time segmentation is given by the smallest amount of HR raw data enabling reliable estimates of the complexity index of each segment. The red dashed lines provide an example of how the total variation is computed measuring the "strengths of the complexity changes of the heart rate" [15]. In the case of the presented data, the total variation is equal to 1.37 . Thus the adaptability is approximately equal to 0.73 .

$$
\begin{aligned}
& \text { Adaptability } \stackrel{\text { def }}{=} \text { a function of (Intrinsic Reactivity Recorded by } \\
& \text { Sensory Surrogate Data), } \\
& \text { Congruency } \stackrel{\text { def }}{=} \text { a function of (Adaptability), } \\
& \text { CSSI } \stackrel{\text { def }}{=} \text { Spectral Distance to a Reference Congruency Matrix. }
\end{aligned}
$$

\subsection{Adaptability}

We define adaptability as the product of the total variation of uniform segments of complexity measures of self-similar normally distributed physiological and behavioural data multiplied by the frequency of this input. The frequency is given by the reciprocal of the count of uniform segments of time considered. The total variation, given in one dimension by arc-length, is denoted $T V$. The complexity measure is given by the Hurst index [16]. The total variation of the considered segmented physiological and behavioural data is given by

$$
\operatorname{TV}(H(\mathcal{Z}))=\max _{\{i=1, \cdots, m\} \in \mathcal{P}[0, T]} \sum_{i=1}^{m} \|\left[H\left(\mathcal{Z}\left(t_{i}\right)\right) \| \mid\right.
$$

In the above expression $\left\{\mathcal{Z}\left(t_{i}\right), i=1, \cdots, m\right\}$ forms subsequent time partitioning of the surrogate data, assuming that $(0, T)=\bigcup_{i=0}^{m-1}\left(t_{i}, t_{i+1}\right), t_{0}=0$, $t_{m}=T, \mathcal{P}[0, T]$ is the set of all possible such partitioning, and $\llbracket \rrbracket$ denotes a jump of the enclosed quantity. $H\left(\mathcal{Z}\left(t_{i}\right)\right)$ is the Hurst index of the sensory 
surrogate data corresponding to the time interval $\left(t_{i}, t_{i+1}\right)$.

Adaptability is defined by

$$
v(H(\mathcal{Z}))_{T} \stackrel{\text { def }}{=} \mathrm{TV}(H(\mathcal{Z})) \mathrm{f}(H(\mathcal{Z}))
$$

where the frequency of the jumps in the complexity of the data, $f(H(\mathcal{Z}))_{T}$, is defined by

$$
\mathrm{f}(H(\mathcal{Z}))_{T} \stackrel{\text { def }}{=} \frac{1}{T} \operatorname{card}\{\llbracket H(\mathcal{Z}(t)) \rrbracket \mid t \in(0, T)\}
$$

The frequency is simply a number of data samples we consider over a certain period of time. We consider one sample encapsulating two hours of surrogate data acquisition in the presented results. The reason we consider larger time periods than an hour is that we need a sufficient amount of data to estimate the complexity indices.

We use frequency considering that different processes have different timescale characteristics. An example are heart rate and skin temperature time-series, the changes being much faster for heart rate compared to skin temperature.

We measure the strength of jumps of the Hurst exponent computed from the segments of the acquired time-series using total variation. Such a measure has no units. Considering the strength of the jumps projected on one of the $y$-axes we consider the range of a given measure applied to a given time-series. We express the units of total variation of complexity measures, given by (1), in terms of the complexity measure range.

The total variation disregards the sign of jumps. Nevertheless it does indicate the complexity travels on the y-axis. Figure 3 is for illustration purposes of congruency only. The simplified version encoded in (3.2) accounts for the strength of the jumps of the two different complexities that should be about the same if demand is met through the corresponding response. This is the meaning of the construct. This approach is weaker to that shown at Figure 3 where the signs of jumps are highlighted.

Adaptability is designed to measure, using certain properties of surrogate time-series, the capabilities of a given system to adjust the complexity of their
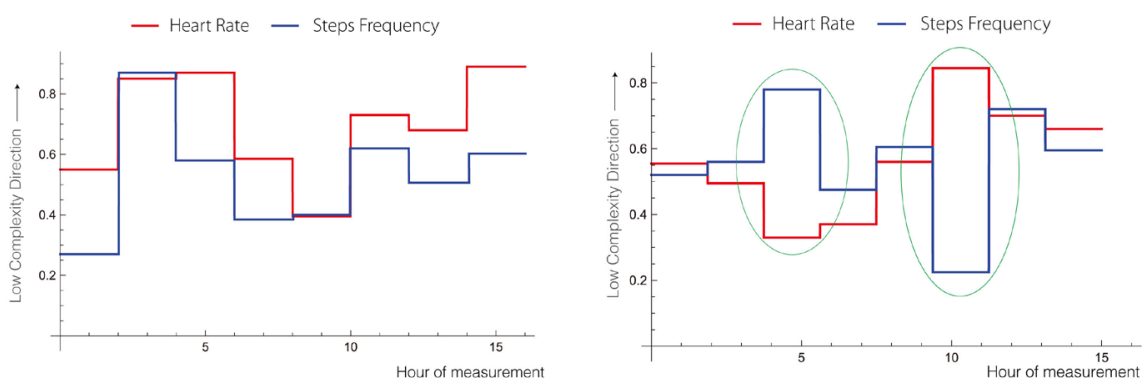

Figure 3. Heart rate and step frequency complexity indices congruency. Left: highly trained healthy subject exhibit congruent characteristics during a typical day. Right: healthy subject exhibits temporal lack of congruent complexity patterns at some time periods (circled). 
physiological and behavioural patterns. We can always assume that the total acquisition time, $T$, is finite, and that the number of sampling equidistant subintervals is non-zero and would be bounded in real data collection. Consequently, it is the total variation that plays the fundamental role.

Assume, given acquired one dimensional sensory data $\mathcal{Z}$,

$$
\operatorname{TV}(H(\mathcal{Z}))=0 \text {, for all partitions given by } \mathcal{P}[0, T], T>0 \text {. }
$$

Given (4) holds, the complexity of the data has to be constant regardless of the number of samples we analyse, i.e., regardless of the time resolution we apply. This implies, almost surely, that $H\left(Z_{i}\right)$ is constant for any $i=1, \cdots, m$. This would imply only two possibilities. Either the system producing $\mathcal{Z}$ does not register any stressors because of the lack of them or it cannot react to demands, assuming the surrogate data chosen are adequate. The former circumstances are unlikely, the latter circumstance implies that the Hausdorff-Besicovitch dimension of $\mathcal{Z}$ is equal to one and that is incompatible with the existence of a living organism.

Assume on the other hand that we increase the frequency but the adaptability stays bounded. That would imply that the total variation has to decrease proportionally to the frequency. Consequently, the variations in the complexity measure with respect to the increasingly higher resolution cannot change much. This is a pronunciation of self-similarity of the sensory data $\mathcal{Z}$. These considerations lead to the multiplicative structure of the adaptability definition (2).

Summarising the two above arguments it seems plausible to assume that the higher the reactivity expressed by a higher adaptability index the higher the chances of a leaving organism to deal with demands and to provide the corresponding responses.

\subsection{Stress Coefficient}

A characterisation of demand/response requires at least two players as soon as the system has to respond to a variety of qualitatively different stressors. An example: a requirement of higher steps frequency must be accompanied by higher breathing rate as well as higher heart rate. The stress coefficient we introduce below is a dimensionless renormalisation constant relating adaptivity measures of two, possibly similar/correlated, surrogate variables.

We choose to use all the available data regardless of their implicit characterisation.

Consider two acquired approximately self-similar time-series, $\mathcal{X}$ and $\mathcal{Y}$. The stress coefficient, $\sigma(\mathcal{X}, \mathcal{Y})$, for the sensory data $\mathcal{X}, \mathcal{Y}$, is given by,

$$
v(H(\mathcal{X}))=\hat{\sigma}(\mathcal{X}, \mathcal{Y}) v(H(\mathcal{Y}))
$$

where $v(H(\cdot))$ is given by (2) and $H$ denotes the Hurst exponent. Notice that stress renormalization constant is not symmetric, i.e., $\hat{\sigma}(\mathcal{X}, \mathcal{Y}) \neq \hat{\sigma}(\mathcal{Y}, \mathcal{X})$. Since 


$$
\hat{\sigma}(\mathcal{X}, \mathcal{Y})=\hat{\sigma}(\mathcal{Y}, \mathcal{X})^{-1}
$$

then, $\hat{\sigma}(\cdot, \cdot)>0$,

$$
|\log (\hat{\sigma}(\mathcal{X}, \mathcal{Y}))|=|\log (\hat{\sigma}(\mathcal{Y}, \mathcal{X}))| .
$$

Thus we implement

$$
\sigma(\mathcal{X}, \mathcal{Y}) \stackrel{\text { def }}{=}|\log (\hat{\sigma}(\mathcal{X}, \mathcal{Y}))|
$$

as the stress coefficient for the reason of symmetry.

Two examples of various stress coefficients given by (5) are presented by Table A4 and Table A5 in Appendix.

\subsection{Congruency}

Suppose a stress coefficient $\sigma$ is equal to one. This can occur if and only if the underlying surrogate time-series $\mathcal{Y}$ and $\mathcal{X}$ have the same distributions. The reason is that for the stress coefficient to be equal to one both sums of the jump magnitudes of the Hurst exponents must equate. Assume further that both processes have the same data acquisition frequency. These processes posses ideal congruency. Surely, congruency is unattainable by human physiological and behavioural patterns. However, we use this construct to obtain a fixed point against which we can measure stress corresponding to various physiological and behavioural patterns.

It follows from (5) that $\sigma(\mathcal{X}, \mathcal{Y})>1$ if the adaptability of the process $\mathcal{X}$ is higher than that of $\mathcal{Y}$ and smaller than one in the opposite case.

\section{Context of Congruency}

What precedes deserves some further comments and interpretation. We mentioned that the lack of temporal congruency may indicate that the adaptability of the controlling response may be inadequate given the imposed demands. However, this may not be entirely true. Let us consider some more extreme acute stress reactions, i.e., fight-or-flight scenario, to be adequate reactions. In these stress situations, the individual is likely to show congruency of complexity patterns. However, this is true only as long as we consider the adequate parameters, meaning orthosympathetic arousal and high physiological and behavioural patterns. This is different in another classical stress situation physiological and behaviourally characterized by freezing with nearly no macroscopic behaviour at all, but with most likely very high orthosympathetic arousal. In an observational sequence containing a freezing episode, this would appear as a major incongruence between complexity of some physiological parameters such as, e.g., heart rate and that of physiological and behavioural parameters such as step frequency. Overall, we may argue that high congruency over time between different physiological and behavioural parameters is a feature of harmony and low physical and psychological stress levels. As a corollary, lower levels of congruency over time or major congruency leaps may be indicative of low harmony or disorder such as chronic stress. 
The congruency concept is illustrated by Figure 3. The left plot indicates congruent structure while the other one does not. Congruency indicates that when complexity of a demand increases the complexity of some related responses should increase as well. Time-local lack of congruency indicates complexity of the controlling response is inadequate relative to the imposed demands. This is shown at the left graph. The graph shown on the right indicates temporal dis-congruencies. At around the fifth hour of the sensory data acquisition the complexity of steps frequency is very low while the complexity of the heart rate is very high. Five hours later the relation of the complexities is reversed. In other words, the congruency accounts for the fact that a positive jump in the complexity of demand is or is not accompanied by the positive jump in the complexity of the corresponding response and vice versa. The circled complexity characteristics exhibit just the opposite. Considering just heart rate/steps frequency is likely an oversimplification, though. An individual actively escaping a danger could increase both heart rate and steps frequency simultaneously in a congruent manner. It is plausible to assume that over a longer period of time such a singular event can be filtered out to obtain a more stable longitudinal congruency image that might be characteristic for a given individual.

\subsection{Compound Spectral Stress Index}

The Compound Spectral Stress Index (CSSI) is constructed to achieve two objectives: 1) to compress stress coefficients tables information, e.g., Table A4 and Table A5, into a single number, and 2) to provide a non-biased demandresponse distance from the ideal congruency. These objectives are addressed using the spectral norm of the covariance matrix difference between ideal congruency and a given stress coefficient matrix.

The ideal congruency matrix, $\mathcal{B}$, is given by $\mathcal{B}=\left\{b_{i, j}\right\}_{i, j=1}^{m}$, for some $m \in \mathbb{N}$, where $\mathcal{B}$ is the identity matrix corresponding to assumed absence of stress. The real stress coefficients matrices, $\mathcal{A}$, are constructed to be the upper triangular matrix images of tables such as those shown at Table A4 and Table A5.

We define the Compound Spectral Stress Index, $\tau(\mathcal{A}, \mathcal{B})$, by

$$
\tau(\mathcal{A}, \mathcal{B}) \stackrel{\text { def }}{=} \max \left\{\rho\left((\mathcal{A}-\mathcal{B})^{\mathrm{T}}(\mathcal{A}-\mathcal{B})\right)\right\}
$$

where $\rho$ denotes the spectrum of the enclosed matrix. This means that CSSI is given by the spectral distance of $\mathcal{A}$ from the reference congruency given by the matrix $\mathcal{B}$. The spectral distance, or equivalently the spectral norm, is a reliable metric yielding a distance measure given by the largest singular value of the matrix $\mathcal{A}-\mathcal{B}$, i.e., by the largest eigenvalue of its covariance form used in (7).

It is important to keep the ordering of the sensory sequencing constant to obtain consistent CSSIs.

An example of CSSIs pertaining to eight healthy subjects is given by Figure 4 . 


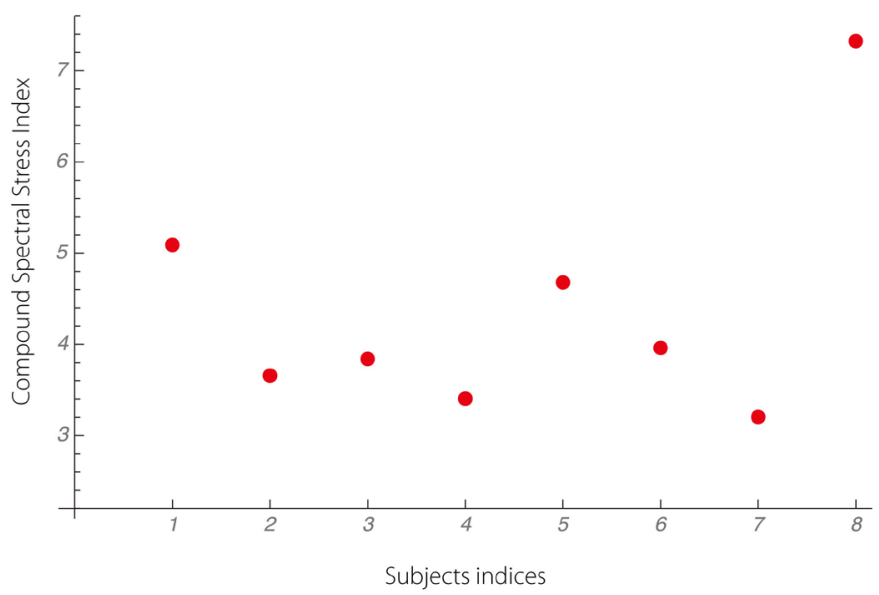

Figure 4. Combined plot of the spectral stress indices of the data based on eight subjects. The plot show different spectral stress indices of eight individuals during exercise, sleep and routine work related to daily activities. The subjects range from highly trained individuals to healthy subjects. The data ware obtained using an arm attached sensor collection data for fifteen hours every thirty seconds. An example of different information we gather from this plot is follows. Subject \#5 experiences a higher stress during extended hours of sleep compared to highly trained subject \#4 with only four hours of sleep. The subjects reporting regular exercise patterns exhibit approximately similar capability to deal with stress-responce patterns as opposed to the subject $\# 8$ who lacks such a pattern. The non-invasive measurements were made in similar environments corresponding to standard daily routines. The sensors have no disruptive physiological and behavioural effects.

The posteriori correlation with questionnaire based subjective self-evaluations suggests that the increase of the CSSI corresponds to the decreasing adaptive capacity over a given time frame. The smaller the value of CSSI the higher is the subjet's capability to deal with various forms of stressors.

We show the high variability among normal individuals that is captured by our analyses in real-life situations based on thousands of data points. That is different compared to other studies that use a higher number of individuals, but only just a few data points. The data yielding the CSSIs of the eight individuals are based on the tables shown in Appendix.

\section{Conclusions}

Physiological and behavioural phenomena in psychiatry are characterized by clinical features of different kinds evolving over time. Stress is among the more important physiological and behavioural phenomena in psychiatry. Currently, there is no clinical tool to evaluate stress levels over fine-grained time periods available for mental health professionals. Current stress measures are limited to the use of subjective data and clinically hardly useful occasional measures of stress hormones in the blood. None of the two is a gold standard for stress measures in numerous clinical settings as they are either subjective, i.e., allowing for no reliable follow-up of stress in, say, alexithymics or the epidemiologically growing group of patients with neurocognitive disorders. Similarly, the objective measure at hand do not allow for an adequate follow-up or the changes over 
time in a non-invasive way.

An alternative is introduced in the presented communication. It is based on measuring physiological and behavioural parameters non-invasively over time, and combining them as complexity indicators allowing for congruency comparison between parameters may be a better way forward. Although this seems likely, it has still to been shown, however, that this approach is really superior in clinical settings and for many conditions than simply asking subjects about their stress level.

The presented approach allows for the extraction of complex information that we consider as a possible indicator of stress as it evolves over time. Currently there is no commonly admitted gold standard of how to measure stress objectively or to distinguish various forms of stress, such as the various acute stress responses mentioned, or chronic stress. This differential approach also suggests that a single CSSI may not be sufficient as an indicator of different forms of stress. However, different CSSI can be calculated based on various combinations of surrogate data, once complexity analyses have been carried out. Thus, our approach needs validation in at least two clinical domains.

First, an elaboration is needed to choose the most appropriate CSSIs as indicators of more specific forms of stress. Second, we need to confront our approach to other measure of stress although, as mentioned, there is no established gold standard. Thus, the validation of the presented approach rests with using both subjective data of stress levels collected over time and objective measures of experimentally collected stress-related neurohormones at short time intervals. This approach is opening up a complex field of research that has to show its usefulness in clinical settings and to figure out what might be the best interface between complexity analysis and user-friendly information transfer to clinicians.

\section{Acknowledgements}

The raw data were processed by the Cassiopée computational ecosystem created by PK. The data used in the presented communication were also used in [10] pertaining to a different framework not for constitutive stress modelling purposes.

\section{Content for Publication}

We declare that the presented research was carried out in accordance with the Declaration of Helsinki and CIOM, and that every subject gave his/her written informed consent.

\section{Funding}

Our activities were supported in part by the Service Universitaire de Psychiatrie de l'Age Avancé that is part of Centre hospitalier universitaire vaudois and University of Lausanne, Switzerland.

\section{Ethics Statement}

We declare that the study was carried out in accordance with the Declaration of 
Helsinki and CIOM, and that every subject gave his/her written informed consent.

\section{Author's Contributions}

The presented theory was developed by both authors. The activity leading to the presented results emerged from intertwining of mathematics and psychiatric research.

\section{Conflicts of Interest}

The authors declare that they have no competing interests. PK is the founder and Chairman of Cassiopee Applied Soutions, Sàrl. The company strives to bring original research to market delivering important tools pertaining to emotional workplaces.

\section{References}

[1] McEwen, B.S. (2017) Neurobiological and Systemic Effects of Chronic Stress. Chronic Stress, 1, 2470547017692328.

[2] Liew, W.S., Seera, M., Loo, C.K., Lim, E. and Kubota, N. (2016) Classifying Stress from Heart Rate Variability Using Salivary Biomarkers as Reference. IEEE Transactions on Neural Networks and Learning Systems, 27, 2035-2046. https://doi.org/10.1109/TNNLS.2015.2468721

[3] Hellhammer, D.H., Wust, S. and Kudielka, B.M. (2009) Salivary Cortisol as a Biomarker in Stress Research. Psycho-Neuro-Endocrinology, 34, 163-171. https://doi.org/10.1016/j.psyneuen.2008.10.026

[4] Webster, J.G. (1997) Design of Pulse Oximeters. Series in Medical Physics and Biomedical Engineering. CRC Press. https://doi.org/10.1887/0750304677

[5] Yuan, S., Nguyen, M.H., Blitz, P., French, B., Fisk, S., De La Torre, O., Smailagic, A., Siewiorek, D.P., Al Absi, M., Ertin, E., Kamarck, T. and Kumar, S. (2010) Personalised Stress Detection from Physiological Measurements, 2010. International Symposium on Quality of Life Technology Proceedings.

[6] Wijsman, J., Grundlehner, B., Liu, H., Hermens, H. and Penders, J. (2011) Towards Mental Stress Detection Using Wearable Physiological Sensors. Engineering in Medicine and Biology Society, EMBC, Annual International Conference of the IEEE, 1798-1801. https://doi.org/10.1109/IEMBS.2011.6090512

[7] Yoon, S., Sim, J.K. and Cho, Y.-H. (2016) A Flexible and Wearable Human Stress Monitoring Patch. Scientific Reports, 6.

[8] Bassingthwaighte, J.B., Liebovitch, L.S. and West, B.J. (1994) Fractal Physiology. American Physiological Society: The American Physiological Society Methods in Physiology Series. American Physiological Society. https://doi.org/10.1007/978-1-4614-7572-9

[9] Koolhaas, J.M., Bartolomucci, A., Buwalda, B., de Boer, S.F., Flugge, G., Korte, S.M., Meerlo, P., Murison, R., Olivier, B., Palanza, P., Richter-Levin, G., Sgoifo, A., Steimer, T., Stiedl, O., van Dijk, G., Wohr, M. and Fuchs, E. (2011) Stress Revisited: A Critical Evaluation of the Stress Concept. Neuroscience \& Biobehavioral Reviews, 35, 1291-1301. https://doi.org/10.1016/j.neubiorev.2011.02.003

[10] Kloucek, P., Zakharov, P. and von Gunten, A. (2016) The Compound Indexing of Human Self-Similar Behavioural Patterns. Journal of Applied Mathematics, 7, 2212 
2228. https://doi.org/10.4236/am.2016.717176

[11] Kloucek, P. and von Gunten, A. (2016) On the Possibility of Identifying Human Subjects Using Behavioural Complexity Analyses. Quantitative Biology, 4, 261-269. https://doi.org/10.1007/s40484-016-0088-8

[12] Porges, S.W. (1992) Vagal Tone: A Physiologic Marker of Stress Vulnerability. Pediatrics, 90, 498-504.

[13] Lee, J., Hwang, Y., Cheon, K. and Jung, H. (2012) Emotion-on-a-Chip (eoc): Evolution of Bio Chip Technology to Measure Human Emotion Using Body Fluids. Medical Hypotheses, 79, 827-832. https://doi.org/10.1016/j.mehy.2012.09.002

[14] Cicchetti, D. (2010) Resilience under Conditions of Extreme Stress: A Multilevel Perspective. World Psychiatry, 9, 145-154. https://doi.org/10.1002/j.2051-5545.2010.tb00297.x

[15] Holden, J.G., Riley, M.A., Gao, J. and Torre, K. (2013) Fractal Analyses: Statistical and Methodological Innovations and Best Practices. Frontiers in Physiology, 4, 97. https://doi.org/10.3389/fphys.2013.00097

[16] Van Ness, M. (1968) Fractional Brownian Motions, Fractional Noise and Application. SIAM Review, 10, 422-437. 


\section{Appendix: Stress Coefficient Tables}

The presented and similar tables are transformed into upper triangular matrices, used to compute CSSIs by inserting zeros below the main diagonal to obtain a square matrix.

Table A1. The stress coefficients of subject \#1. The stress coefficients are obtained from 4 equidistant time segments of acquired data encompassing about 15 hours of data acquisition.

\begin{tabular}{ccccccc}
\hline Quantities & $\begin{array}{c}\text { Heart } \\
\text { Rate }\end{array}$ & $\begin{array}{c}\text { Blood } \\
\text { Oxygenation }\end{array}$ & Perfusion & $\begin{array}{c}\text { Skin } \\
\text { Temperature }\end{array}$ & $\begin{array}{c}\text { Relative } \\
\text { Movement }\end{array}$ & $\begin{array}{c}\text { Steps } \\
\text { Frequency }\end{array}$ \\
\hline $\begin{array}{c}\text { Heart Rate } \\
\text { Blood }\end{array}$ & 1 & 0.566519 & 0.3333 & 1.63602 & 0.718639 & 0.973919 \\
Oxygenation & & 1 & 0.58833 & 2.88784 & 1.26852 & 1.71913 \\
Perfusion & & & 1 & 4.90854 & 2.15613 & 2.92205 \\
$\quad \begin{array}{l}\text { Skin } \\
\text { Temperature }\end{array}$ & & & 1 & 0.439262 & 0.595299 \\
$\quad \begin{array}{l}\text { Relative } \\
\text { Movement } \\
\text { Steps }\end{array}$ & & & & 1 & 1.35523 \\
Frequency & & & & & \\
\hline
\end{tabular}

Table A2. The stress coefficients of subject \#2. The stress coefficients are obtained from 5 equidistant time segments of acquired data encompassing about 15 hours of data acquisition.

\begin{tabular}{ccccccc}
\hline Quantities & $\begin{array}{c}\text { Heart } \\
\text { Rate }\end{array}$ & $\begin{array}{c}\text { Blood } \\
\text { Oxygenation }\end{array}$ & Perfusion & $\begin{array}{c}\text { Skin } \\
\text { Temperature }\end{array}$ & $\begin{array}{c}\text { Relative } \\
\text { Movement }\end{array}$ & $\begin{array}{c}\text { Steps } \\
\text { Frequency }\end{array}$ \\
\hline Heart Rate & 1 & 0.436976 & 0.108716 & 0.452929 & 0.149075 & 0.161365 \\
$\quad$ Blood & & 1 & 0.248793 & 1.03651 & 0.341152 & 0.369277 \\
Oxygenation & & & 1 & 4.16615 & 1.37123 & 1.48427 \\
$\quad \begin{array}{c}\text { Perfusion } \\
\quad \text { Skin }\end{array}$ & & & 1 & 0.329136 & 0.35627 \\
$\begin{array}{c}\text { Temperature } \\
\text { Relative }\end{array}$ & & & & & 1.08244 \\
Movement & & & & & \\
$\quad$ Steps & & & & & & 1 \\
Frequency & & & & & & \\
\hline
\end{tabular}

Table A3. The stress coefficients of subject \#3. The stress coefficients are obtained from 3 equidistant time segments of acquired data encompassing about 15 hours of data acquisition.

\begin{tabular}{ccccccc}
\hline Quantities & $\begin{array}{c}\text { Heart } \\
\text { Rate }\end{array}$ & $\begin{array}{c}\text { Blood } \\
\text { Oxygenation }\end{array}$ & Perfusion & $\begin{array}{c}\text { Skin } \\
\text { Temperature }\end{array}$ & $\begin{array}{c}\text { Relative } \\
\text { Movement }\end{array}$ & $\begin{array}{c}\text { Steps } \\
\text { Frequency }\end{array}$ \\
\hline Heart Rate & 1 & 0.260791 & 0.608796 & 0.203685 & 0.743987 & 0.0761693 \\
$\quad$ Blood & & 1 & 2.33442 & 0.781028 & 2.85281 & 0.292071 \\
$\begin{array}{c}\text { Oxygenation } \\
\text { Perfusion }\end{array}$ & & & 1 & 0.33457 & 1.22206 & 0.125115 \\
$\quad$ Skin & & & 1 & 3.65264 & 0.373957 \\
$\begin{array}{c}\text { Temperature } \\
\text { Relative } \\
\text { Movement } \\
\text { Steps }\end{array}$ & & & & & 0.10238 \\
Frequency & & & & & & 1 \\
\hline
\end{tabular}


Table A4. The stress coefficients of subject \#4. The stress coefficients are obtained from 8 equidistant time segments of acquired data encompassing about 15 hours of data acquisition.

\begin{tabular}{ccccccc}
\hline Quantities & $\begin{array}{c}\text { Heart } \\
\text { Rate }\end{array}$ & $\begin{array}{c}\text { Blood } \\
\text { Oxygenation }\end{array}$ & Perfusion & $\begin{array}{c}\text { Skin } \\
\text { Temperature }\end{array}$ & $\begin{array}{c}\text { Relative } \\
\text { Movement }\end{array}$ & $\begin{array}{c}\text { Steps } \\
\text { Frequency }\end{array}$ \\
\hline $\begin{array}{c}\text { Heart Rate } \\
\text { Blood }\end{array}$ & 1 & 1.67598 & 1.92038 & 2.51267 & 0.42252 & 1.68351 \\
$\begin{array}{c}\text { Oxygenation } \\
\text { Perfusion }\end{array}$ & 1 & 1.14583 & 1.49923 & 0.252104 & 1.0045 \\
$\quad$ Skin & & 1 & 1.30843 & 0.220019 & 0.876657 \\
$\begin{array}{c}\text { Temperature } \\
\text { Relative }\end{array}$ & & & 1 & 0.168155 & 0.670009 \\
Movement & & & & & \\
$\quad$ Steps & & & & & \\
Frequency & & & & & \\
\hline
\end{tabular}

Table A5. The stress coefficients of subject \#5. The stress coefficients are obtained from 3 equidistant time segments of acquired data encompassing about 15 hours of data acquisition.

\begin{tabular}{ccccccc}
\hline Quantities & $\begin{array}{c}\text { Heart } \\
\text { Rate }\end{array}$ & $\begin{array}{c}\text { Blood } \\
\text { Oxygenation }\end{array}$ & Perfusion & $\begin{array}{c}\text { Skin } \\
\text { Temperature }\end{array}$ & $\begin{array}{c}\text { Relative } \\
\text { Movement }\end{array}$ & $\begin{array}{c}\text { Steps } \\
\text { Frequency }\end{array}$ \\
\hline Heart Rate & 1 & 4.98645 & 1.96584 & 1.17414 & 2.68461 & 0.708285 \\
Blood & & 1 & 0.394236 & 0.235466 & 0.53838 & 0.142042 \\
Oxygenation & & 1 & 0.597272 & 1.36563 & 0.360297 \\
$\begin{array}{c}\text { Perfusion } \\
\text { Skin }\end{array}$ & & & 1 & 2.28645 & 0.603238 \\
$\begin{array}{c}\text { Temperature } \\
\text { Relative }\end{array}$ & & & & & 0.263832 \\
Movement & & & & & \\
$\quad$ Steps & & & & & & \\
Frequency & & & & & & \\
\hline
\end{tabular}

Table A6. The stress coefficients of subject \#6. The stress coefficients are obtained from 8 equidistant time segments of acquired data encompassing about 15 hours of data acquisition.

\begin{tabular}{|c|c|c|c|c|c|c|}
\hline Quantities & $\begin{array}{c}\text { Heart } \\
\text { Rate }\end{array}$ & $\begin{array}{c}\text { Blood } \\
\text { Oxygenation }\end{array}$ & Perfusion & $\begin{array}{c}\text { Skin } \\
\text { Temperature }\end{array}$ & $\begin{array}{c}\text { Relative } \\
\text { Movement }\end{array}$ & $\begin{array}{c}\text { Steps } \\
\text { Frequency }\end{array}$ \\
\hline Heart Rate & 1 & 2.27671 & 1.58872 & 0.985259 & 0.402659 & 1.82687 \\
\hline $\begin{array}{c}\text { Blood } \\
\text { Oxygenation }\end{array}$ & & 1 & 0.697813 & 0.432756 & 0.17686 & 0.802418 \\
\hline Perfusion & & & 1 & 0.62016 & 0.253449 & 1.1499 \\
\hline $\begin{array}{c}\text { Skin } \\
\text { Temperature }\end{array}$ & & & & 1 & 0.408683 & 1.85421 \\
\hline $\begin{array}{c}\text { Relative } \\
\text { Movement }\end{array}$ & & & & & 1 & 4.53703 \\
\hline $\begin{array}{c}\text { Steps } \\
\text { Frequency }\end{array}$ & & & & & & 1 \\
\hline
\end{tabular}


Table A7. The stress coefficients of subject \#7. The stress coefficients are obtained from 3 equidistant time segments of acquired data encompassing about 15 hours of data acquisition.

\begin{tabular}{ccccccc}
\hline Quantities & $\begin{array}{c}\text { Heart } \\
\text { Rate }\end{array}$ & $\begin{array}{c}\text { Blood } \\
\text { Oxygenation }\end{array}$ & Perfusion & $\begin{array}{c}\text { Skin } \\
\text { Temperature }\end{array}$ & $\begin{array}{c}\text { Relative } \\
\text { Movement }\end{array}$ & $\begin{array}{c}\text { Steps } \\
\text { Frequency }\end{array}$ \\
\hline Heart Rate & 1 & 1.27931 & 0.723648 & 2.3792 & 1.36328 & 1.07331 \\
$\begin{array}{c}\text { Blood } \\
\text { Oxygenation }\end{array}$ & 1 & 0.565655 & 1.85976 & 1.06564 & 0.838974 \\
Perfusion & & 1 & 3.28779 & 1.8839 & 1.48319 \\
$\quad$ Skin & & & 1 & 0.573 & 0.45112 \\
$\begin{array}{c}\text { Temperature } \\
\text { Relative }\end{array}$ & & & & & \\
Movement & & & & & & \\
Steps & & & & & & \\
Frequency & & & & & & \\
\hline
\end{tabular}

Table A8. The stress coefficients of subject \#8. The stress coefficients are obtained from 3 equidistant time segments of acquired data encompassing about 15 hours of data acquisition.

\begin{tabular}{|c|c|c|c|c|c|c|}
\hline Quantities & $\begin{array}{l}\text { Heart } \\
\text { Rate }\end{array}$ & $\begin{array}{c}\text { Blood } \\
\text { Oxygenation }\end{array}$ & Perfusion & $\begin{array}{c}\text { Skin } \\
\text { Temperature }\end{array}$ & $\begin{array}{c}\text { Relative } \\
\text { Movement }\end{array}$ & $\begin{array}{c}\text { Steps } \\
\text { Frequency }\end{array}$ \\
\hline Heart Rate & 1 & 1.81883 & 6.96364 & 3.11701 & 0.388888 & 0.383757 \\
\hline $\begin{array}{c}\text { Blood } \\
\text { Oxygenation }\end{array}$ & & 1 & 3.82864 & 1.71375 & 0.213812 & 0.210991 \\
\hline Perfusion & & & 1 & 0.447613 & 0.0558456 & 0.0551087 \\
\hline $\begin{array}{c}\text { Skin } \\
\text { Temperature }\end{array}$ & & & & 1 & 0.124763 & 0.123117 \\
\hline $\begin{array}{c}\text { Relative } \\
\text { Movement }\end{array}$ & & & & & 1 & 0.986805 \\
\hline $\begin{array}{c}\text { Steps } \\
\text { Frequency }\end{array}$ & & & & & & 1 \\
\hline
\end{tabular}

